\title{
The Design and Research of the Control System of Automatic Intelligent Rice Cooker, based on STM32
}

\author{
Hai-qiang Zhao* ${ }^{1}$, and Han Zhao ${ }^{2}$ \\ ${ }^{1}$ Tianjin Polytechnic University, Tianjin, China \\ ${ }^{2}$ Tianjin Polytechnic University, Tianjin, China \\ ${ }^{*}$ Corresponding author
}

Keywords: STM32, Intelligent Rice Cooker, Remote Control, Speech Recognition, Environmental-friendly.

\begin{abstract}
This automatic intelligent rice cooker is designed on the base of STM32.It consists of automatic rice supply, rice washing and cooking functions, which can be realized, in the long distance, by sending messages, using smart phone apps, Bluetooth or other remote control devices.[1]In the meantime, the rice cooker would send the working status as feedback signal to users. The user also can use the automatic speech recognition and touch panel technique. Different sensors are used on the real time surveillance of the quantity of rice and water level, so that the cooker will alarm no matter when the quantities of rice and water are abnormal. For the environmontal-friendly and energy saving issues, we take use of ultra filtration to help filter the waste water after the rice washing process. This automatic intelligent rice cooker is designed mainly for students and office workers and provides a time-saving solution for them to have tasty rice.
\end{abstract}

\section{Introduction}

In the recent years, with the development of mobility internet and the popularization of the smart phone, the electric devices become more and more intelligent, and some of them have already changed the people's lifestyle, significantly. Comparing with the traditional electric devices, intelligent electric devices perform far better on satisfying users' requirements towards the ideal of life. From the market research, what can be easily found is that most of the rice cookers need to be set manually and lack of remote control functions. The result of the research shows the low intelligence of rice cookers. Therefore, our automatic intelligent rice cooker is able to overcome the shortcomings of traditional rice cooker and improve the convenience of rice cooker. Our design, in the meantime, uses the ultra filtration membrane to realize the recycle of water, which also meet the energy-saving concept.

\section{The Overall Design of Intelligent Electric Rice Cooker}

Intelligent rice cooker contains mechanical and control system which are shown in figure 1-a.Mechanical system consists of rice supply device, rice washing device, water recycle device, rice cooking device which are shown in figure 1-b. The rice supply device is in charge of calculating the amount the rice and supply it to the pot. Water recycle device works on supplying and draining away water in the rice washing process. As for the rice washing device, it stirs and washes the raw rice. And rice cooking device is in charge of starting the cooking switch.

Control system uses the flush bonding design, which can be divided into software and hardware platform. Software platform is made by controller, message module, voice module, Bluetooth module, touching panel module and power supply module. And software platform consists of embedded operation system and software. Users can send commands via messages. After the microprocessor receives the commands, the motor driven by driving device will operate the cooker to choose the amount of rice, supply water, wash rice, drain out water and cook. Then the relevant operating command would be sent out. In the short distance, users are able to send operating commands through 
touch panel, speech recognition, as well as Bluetooth. This device uses leak-proof sealing structure, so that what can be guaranteed that there would not be any leakage in our machine. Ultra filtration membrane can guarantee that the rice washing water can be collected and recycled for many times, then improve the usage efficiency of the water.

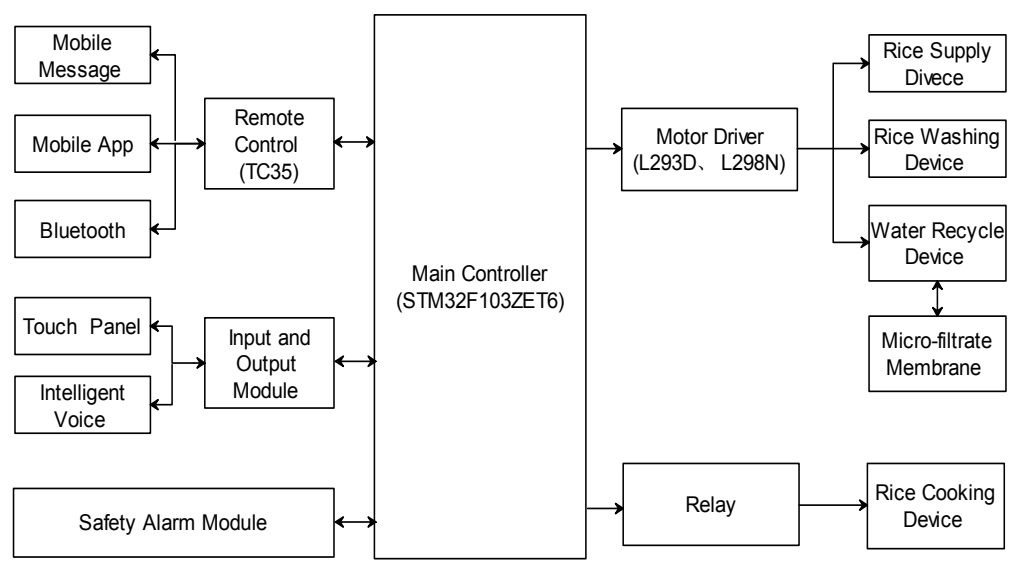

Figure 1-a Overall design of control system

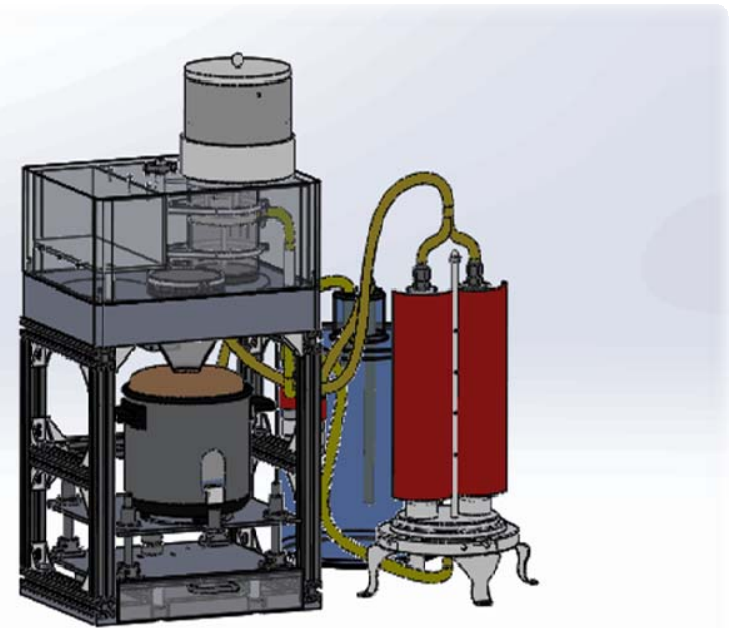

Figure 1-b Mechanical system diagram

\section{Hardware Platform Design}

The hardware part (shown in figure 2) of this device is made by microprocessor system (shown in figure 3), message module, voice module, Bluetooth module, touch panel module, power supply module. Each module is operating with the control of STM32, in order to realize the whole procedures.

\section{Main Control Chip}

STM32F103ZET6 is a 32 bits,cortex-M3 framework chip, with the maximum working frequency of $72 \mathrm{MHz}$ and a $512 \mathrm{~Kb}$ flash memory. Multipath PWM output is required to control the cooker.STM32F103ZET6 is able to export multipath PWM, in the meantime, the real time data will be transmitted and show on the screen through FSMC bus. The rice cooker uses message, Bluetooth 
and app to communicate, send and receive data via ports. There exists three ports on the STM32F103ZET6 chip to satisfy multiple ways of communication. Circuits is shown in figure 3.

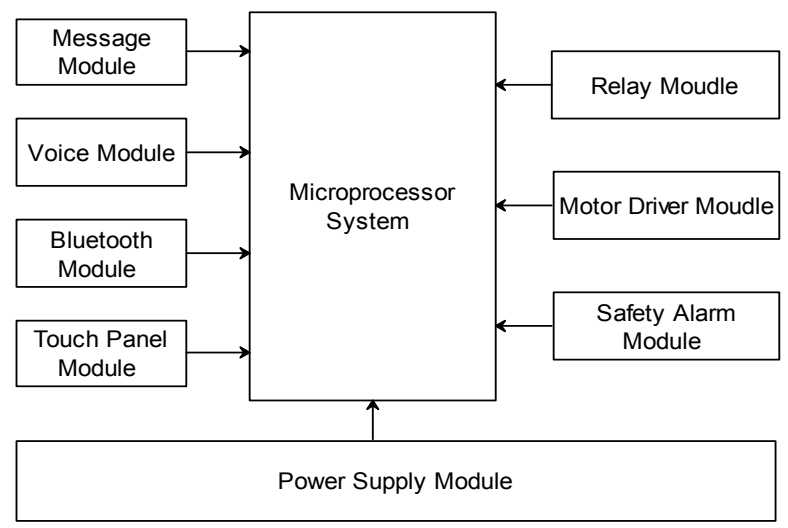

Figure 2 Hardware part

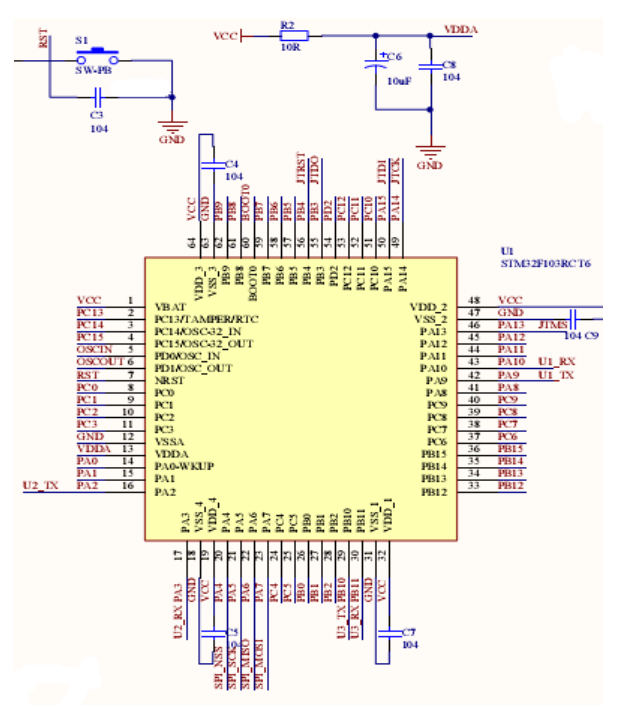

Figure 3 Circuit of the control chip

\section{Message Module}

In this design, rice cooker need receive the remote signal, sent from mobile phone. The user sends message as the control signal, to the cooker. Then after the command has been received, the cooker can wash and cook the rice, with the control of microprocessor. Besides, the microprocessor can also send the feedback signal to user, so that users are able to monitor the working status of their cooker, in case the quantities of both water and rice are abnormal. To realize remote control function, microprocessor can be used to send and receive messages. The commands in common use are shown in table 1. 
Table 1 Common AT command table

\begin{tabular}{lll}
\hline Function & Common & Introduction \\
\hline Connect & AT & TC35 is connected: OK \\
Register network & AT + COPS? & Query the network provider \\
Time & AT + CCLK? & Query the current time \\
Hang up & ATH & Hang up \\
Dial & ATD & Dial the phone number \\
Notification & AT + CNMI & Select the prompt mode \\
Read message & AT + CMGR & Read message \\
Delete message & AT + CMGD & Delete message you store \\
Message format & AT + CMGF & 1-TEXT;0-PDU \\
Send message & AT + CNGS & Send message \\
Set address & AT + CSCA & Set the message center address \\
\hline
\end{tabular}

\section{Voice Module}

LD3320 voice module is used for the automatic speech recognition. To control the cooker, commands require practicability and accuracy. Users are able to use spelling to realize dynamic edit of the LD3320 command, which provides high convenience, practicability and accuracy and makes it easy to identify the statements, like rice-washing and cooking. What's more, this module can also recognize different accents, and provide high convenience to users in different areas. Using this voice module can realize the communication between users and cooker. Speech commands are shown below in table 2.Circuit is shown in figure 4.

Table 2 Voice commands

\begin{tabular}{|c|c|}
\hline Instructions & Function \\
\hline taomi & wash rice \\
\hline fenshu & count \\
\hline yifen & one \\
\hline 1iangfen & two \\
\hline sanfen & three \\
\hline baofan & start \\
\hline qufan & get rice \\
\hline
\end{tabular}

\section{Bluetooth Module}

Bluetooth module is mainly used to receive signal from the phone. High reaction speed is necessary, especially in a short distance communication. What's more, large quantities of data, high reaction time for Bluetooth module and easy matching are required, at the same time. Therefore, even when the signal condition is too poor to send the messages, users can use used to communicate with cooker, as an alternative. Circuit is shown in figure 5. 


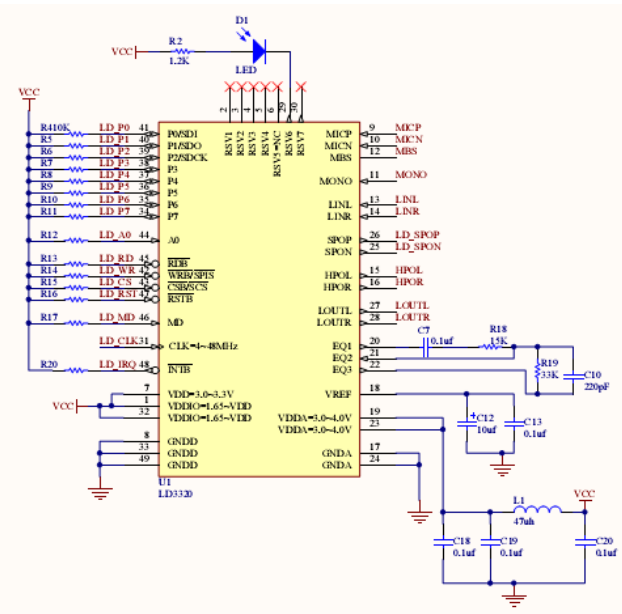

Figure 4 Circuit of voice module

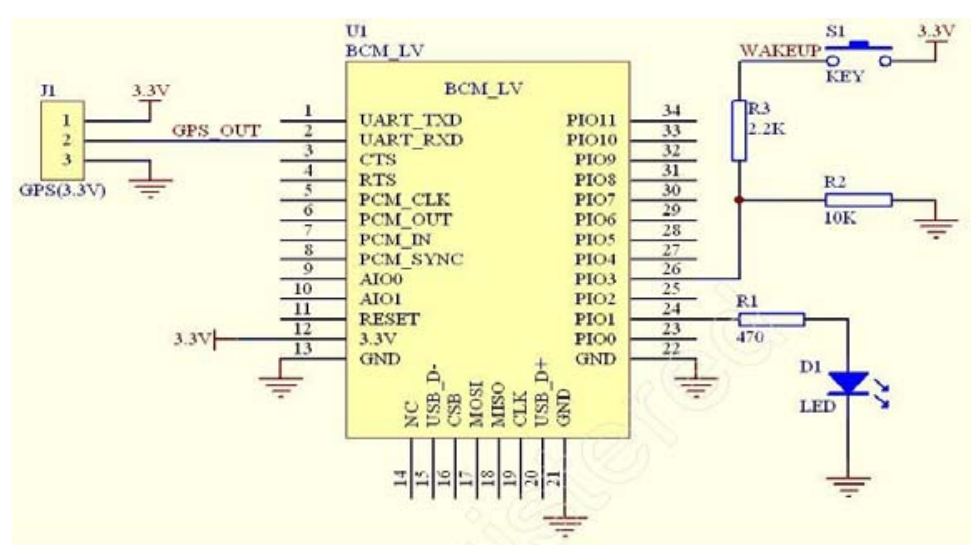

Figure 5 Bluetooth module circuit

\section{Display Module}

The display module uses FSMC $320 * 240$ pixel resistive screen, driven by bus and equipped with touch panel. Resistive screen performs better on anti-interference. With the FSMC bus on STM32,it's cheap and easy to match. Therefore, this screen provides a good experiences on man-machine communication. The display sample is shown in figure 6.

\section{Power Supply Circuit}

Main power supply uses $12 \mathrm{~V} / 2 \mathrm{~A}$ as input supply. And uses standard PW-2.5 port. It can provide 12VDC, 5VDC, 3.3VDC.Due to the LM7805 regulation chip, voltage can be transformed into $5 \mathrm{~V}$, and transferred to driver, Bluetooth module, display module, etc. In the meanwhile, by using AMS1117,voltage can be transformed into 3.3V, as the power supply to CPU and other chips.Power supply circuit is shown in figure 7. 


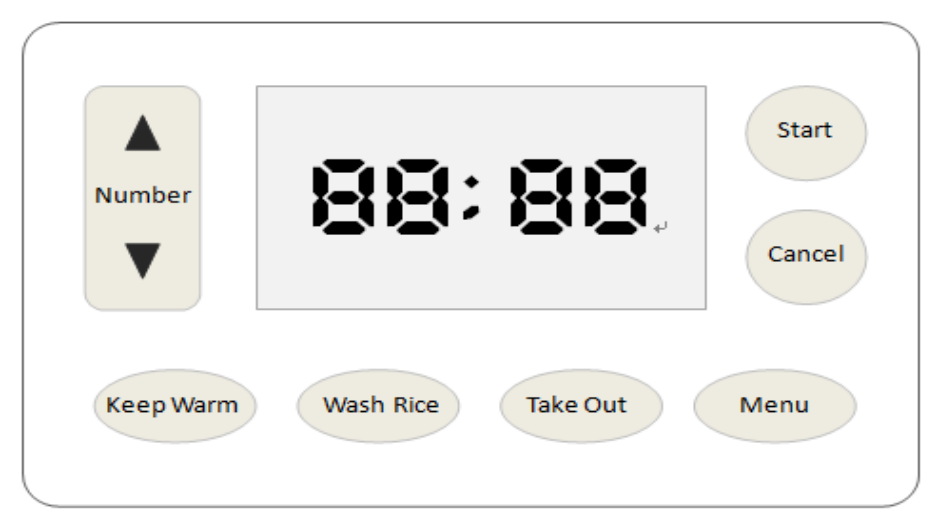

Figure 6 Display module circuit

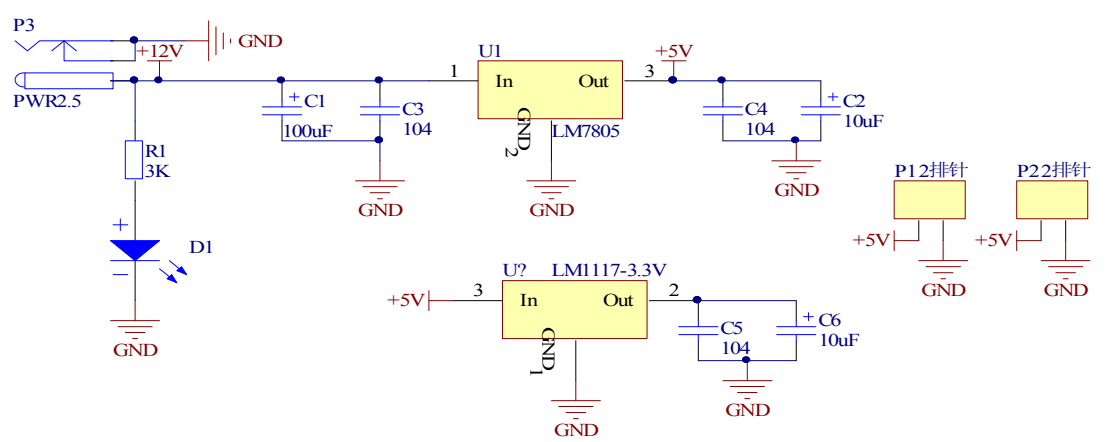

Figure 7 Power supply circuit

\section{Software Platform Design}

Software part is the core factor to control the whole device. It divides different tasks and distributes them to different modulus. Based on the analysis of whole working process, embedded system is used to design software. Each module is responsible for a specific task, which is called to execute the task. Software part consists of signal-receiving part and calling part. The sources of electronic signal are from message, touch panel, Bluetooth and voice. Calling part is in charge of the control of different motors used in rice supply and rice washing processes. The overview of software flow chart is shown in figure 8 .

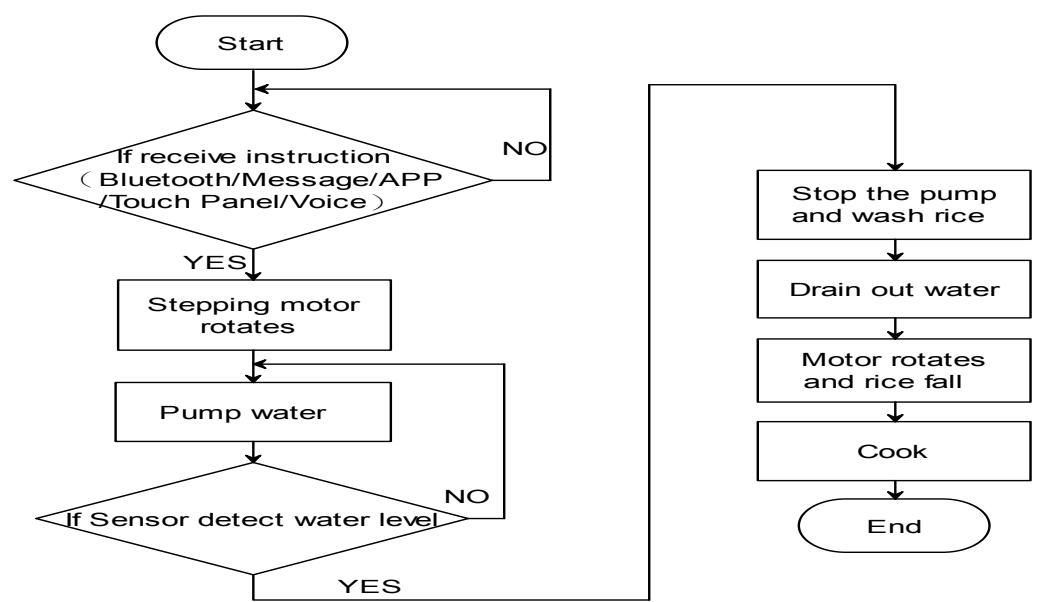

Figure 8 Software flow chart 


\section{Summary}

Automatic intelligent rice cooker combines automatic rice supply, washing and cooking, three functions together. Not only realizing the multiple ways of control, in the meantime, it propagates the environmental friendly concept with water recycle method, fill in the gap of the market. With the faster pace of life, people are trying to get rid of the traditional method, which is that cooking can be only achieved at home. Automatic intelligent control will develop the quality of human's life. Web of things can play an important role in the future. This cooker is following the pace of the initial stage of internet of things. In addition, embedded operation system has great expansion characteristic, which means, it provides a convenient to modify the product, no matter for design itself and technical aspects. I also hope that, with the popularization of the intelligent products, people would adequately enjoy the convenient web of Internet of things Era.

\section{References}

[1]Tong Yao-nan,Chen Song,Li Chun-lai.Design of smart home wireless supervision system based on GPRS[J].Journal of Human Institute of Science and Technology:Natural Science,2011,24(1):72-75.

[2]LI Shu-liang,Chen Chao,XIE Zhao-hong.Design and implementati of real-time wireless video surveillance system based on embedded system[J].Journal of Wuhan Polytechnic University,2010,29(4):49-53.

[3]Nissan Motor Co Ltd,Hitachi Ltd.Energy management system utilizing the discharging of $\mathrm{EV}[\mathrm{R}] .2010$.

[4]Zeng Song-wei,Zhang Yun,Qiu Wei-qiang.Design of intelligent home control system based on Internet of things[J].Modern electronic technology,2011(09):168-171.

[5]Zhao Rui-fen.A preliminary study on the intelligent home of the Internet of things[J].Science and technology information.2010(22):199. 